\title{
Research on Production Process Parameter Management Based on MES System
}

\author{
Zheng Chao, Wang Yan, Zhang Guangshen \\ Information and Software Development Center, Beijing Institute of Aerospace Control Devices, Beijing, China
}

\section{Email address:}

bitstzc@163.com (Zheng Chao)

\section{To cite this article:}

Zheng Chao, Wang Yan, Zhang Guangshen. Research on Production Process Parameter Management Based on MES System. Science Discovery. Vol. 9, No. 2, 2021, pp. 79-84. doi: 10.11648/j.sd.20210902.20

Received: March 17, 2021; Accepted: April 22, 2021; Published: April 26, 2021

\begin{abstract}
It is very important to realize the information management of product parameters in the production workshop, which can timely, accurately, clearly and clearly display the real-time parameter data in the production process, and ensure the efficient cooperation of various departments, so as to improve the processing efficiency and product processing quality of the production workshop. In this paper, the MES system of a military machinery manufacturing enterprise is used as the research platform to collect the product process data of the workshop and form the product parameter library of the production process of the workshop, taking the parameter library as the research object, a parameter management mechanism suitable for mechanical manufacturing enterprises is established to carry out efficient parameter management. The parameter management based on MES system consists of quality card management, parameter management, parameter optimization, parameter filling and verification, and parameter analysis. Neural network and other methods are used to optimize the selection of product parameters and analyze the historical product parameters. The application results show that the parameter management mechanism proposed in this paper can realize the effective management of the production process parameters of the manufacturing enterprise workshop, improve the processing efficiency and product quality, and has a certain reference value for the manufacturing enterprise production process parameter management.
\end{abstract}

Keywords: Parameter Management, Manufacturing Enterprises, Production Process, Process Parameters

\section{基于MES系统的生产过程参数管理研究}

郑超, 王岩, 张广申

信息化与软件开发中心, 北京航天控制仪器研究所, 北京, 中国

邮箱

bitstzc@163.com（郑超）

摘要：实现生产车间产品参数信息化管理, 能够及时、准确、清晰、明了展示生产过程中的实时参数数据, 确保 各个部门高效协同开展工作，对于提升生产车间提高加工效率及产品加工质量等工艺指标至关重要。该文以某军 工类机械制造企业生产车间MES系统为研究平台, 对车间产品工艺数据进行采集, 形成生产车间生产过程产品参数 库, 并以该参数库为研究对象, 建立适合机械制造企业的参数管理机制, 进行高效参数管理。基于MES系统的参数 管理由质卡管理、参数管理、参数优选、参数填报及校验、参数分析五个部分组成。采用神经网络等多种方法进 行产品参数优化选取及历史产品参数分析。应用结果表明, 本文提出的参数管理机制可实现对于制造企业生产车 间生产过程参数的有效管理, 提高了加工效率, 提升了产品质量, 对于制造企业生产过程工艺参数管理具有一定 的参考价值。 
关键词: 参数管理, 制造企业, 生产过程, 工艺参数

\section{1. 引言}

随着中国制造2025战略规划及德国“工业4.0”的提出, 未来制造业将变成一种网络化、信息化、自动化和智能化 相结合的智能工厂 $[1]$ 。为提升企业竞争力, 提高生产管理 能力, 国内制造企业生产车间逐步引入多样化的智能化管 理工具如MES、ERP等。随着生产应用的逐步深入，不少 企业面临生产过程工艺参数管理效率低下的问题。而以信 息化手段实现生产车间产品参数高效管理, 能够及时、准 确、清晰、明了展示生产过程中的实时参数数据, 确保各 个部门高效协同开展工作, 对于提升生产车间提高加工效 率及产品加工质量等工艺指标至关重要。为切实解决部分 生产车间存在的参数管理困难的问题, 本文以某军工类制 造企业生产车间MES系统为研究平台, 针对车间产品工艺 数据进行采集, 建立生产车间产品参数库, 同时利用神经 网络等方法对于产品参数进行优化选取, 进行产品参数分 析, 运用多种智能化手段对于参数进行管理, 建立适合生 产车间的参数管理机制, 在提升生产车间参数管理能力的 同时, 对于同类制造企业生产过程工艺参数管理具有一定 的参考价值。

\section{2. 制造企业生产现状及MES系统概述}

该生产车间所涉行业为工业仪器仪表生产行业。工业 仪器仪表行业是机械工业的重要分支属于典型离散制造 业,具有"多品种、小批量"生产组织模式的难点[2]。离散 制造的产品由各零组件组成, 根据BOM经过一系列不连 续加工工序生产，最终装配而成。不同批量的多项生产任 务并行安排在生产计划中, 执行过程中呈现离散执行特点, 现场与技术管理部门的信息沟通缺少渠道, 离散产品带来 工艺文件更大的离散性, 进一步加剧了在制品的离散性。 上文所涉 $\mathrm{BOM}$ 又被称为物料清单系统, 主要用于描述组 成产品的零部件之间关系的信息, 可以直观地体现出物料 结构的关系, 贯穿于制造业产品生命周期的各个阶段, 是 制造业的核心数据[3]。

MES系统（制造企业生产过程执行系统）的出现为生 产车间管理带来了新的思路。美国先进制造研究机构 (AMR)对MES的定义为:位于上层的计划管理系统与底层 的工业控制之间的面向车间层的管理信息系统,它为操作 人员/管理人员提供计划的执行、跟踪以及所有资源(人、 设备、物料、客户需求等)的当前状态[4]。其功能结构图 如图1所示。该系统能够从产品生产工单发出开始, 一直 到成品产出, 为各项生产管理活动的开展提供信息沟通平 台, 确保生产管理信息得到顺利传递。它通过计划监控, 生产调度, 实时传递生产过程数据, 来处理应对生产制造 过程中的各项复杂问题 [5]。因此在生产管理方面, MES 系统属于信息传递的桥梁, 能够用于实现生产过程的优化 管理, 凭借准确数据为车间生产提供指导。利用系统进行 生产信息录入, 能够避免反复进行信息的抄写, 使人为错
误得到减少 [6]。在生产数据统计分析过程中,利用系统提 供的准确资料可以进一步实现生产工艺流程的优化,为产 品质量检测和售后服务提供详细信息,因此能够使企业生 产管理水平得到提高[6]。

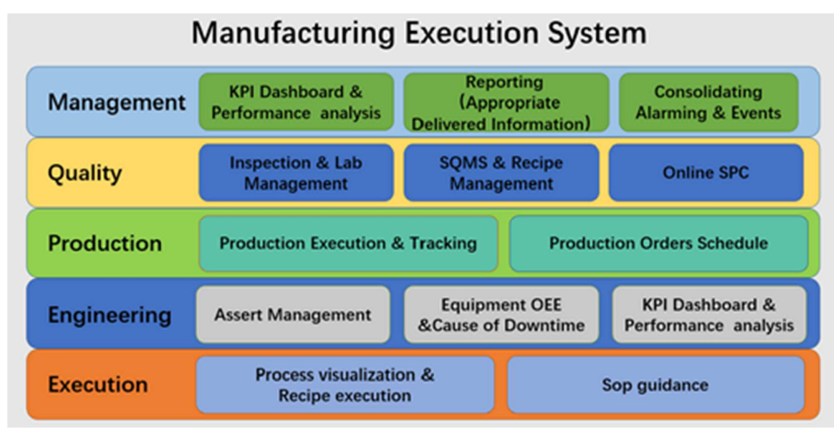

图1 MES系统功能结构图。

\section{3. 基于MES系统的参数管理}

参数管理的功能是收集、处理产品加工过程中各种有 用的工艺信息，为使用者提供相关服务。基于MES系统的 参数管理由质卡管理、参数管理、参数优选、参数填报及 校验、参数分析五个部分组成。参数管理功能结构图如图 2所示, 下文将对所涉功能模块进行详细介绍。

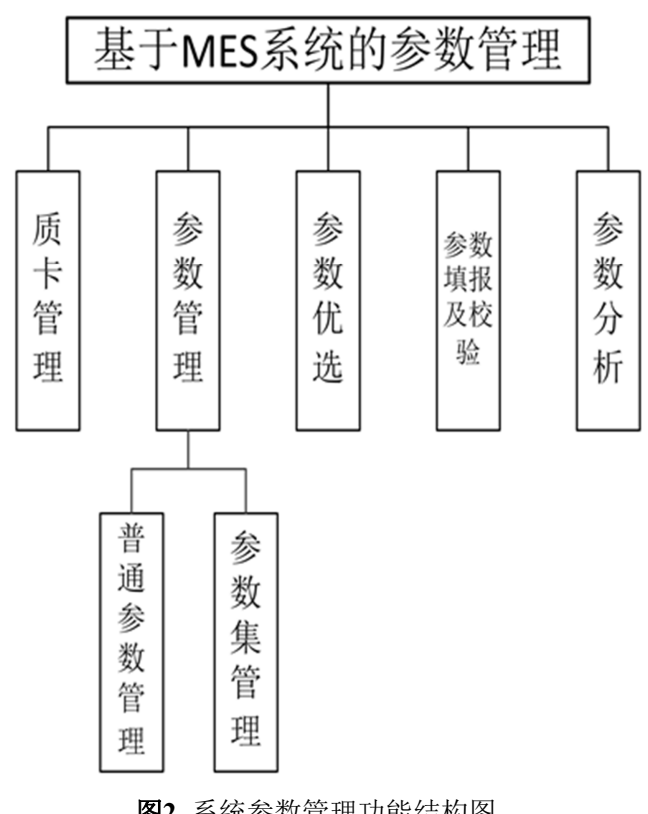

3.1. 质卡管理

\subsection{1. 质量跟踪}

质量跟踪又称产品跟踪, 是目前国内外广泛采用的一 种质量管理方法 [7]。根据ISO国际标准化委员会在质量管 理和质量保证标准中给出的可追溯的定义, 产品跟踪是指 
对产品在业务链中的历史和控制信息进行记录的方法 [8]。 该军工企业生产车间采用精细化的质量管理方案, 运用质 卡等工具记录产品质量信息完成质量跟踪过程, 构建完整 的质量管理链条。精细化质量管理既具有工程技术的属性, 又具有管理理念和方法的属性, 是对两者进行交叉融合形 成的综合性技术成果 [9], 是国内众多制造企业首选的质量 管理方案。

\subsection{2. 质卡定义}

质卡即质量跟踪卡，是国内外生产企业广泛采用的一 种全面、系统地收集和整理产品质量信息的工具，用于记 录产品生产过程中的工序、工步、检查项、零件、原材料、 附表等基础信息，即产品生产过程中的各项参数数据均记 录在质卡中, 这种质量管理模式能够实现产品生产过程数 据可追溯, 对于生产过程进行连续、系统、有效的控制。

\subsection{3. 质卡管理模式}

该军工企业生产车间采用质卡模板-质卡实例的管理 模式。生产车间生产的某一类产品对应同一个质卡模板, 质卡模板示例如图3所示。质卡实例是质卡模板的具体化 表现即每一个产品实体对应一个质卡实例, 生产计划下发 后系统根据该产品对应的质卡模板自动生成相应的质卡 实例, 通过质卡实例的流转对产品的整个生产过程数据进 行跟踪及监控。

质卡模板管理主要实现质卡模板新增、版本控制、删 除、查看、查询、质卡实例生成等功能。系统能够进行严 格的状态监控, 质卡模板由工艺管理人员审批通过后, 方 可进入正式启用状态, 同时, 只有处于启用状态的质卡才 能进行质卡实例生成。质卡实例管理包含质卡实例内容填 报、质卡导出、打印等功能, 质卡实例列表如图4所示。 生产过程所涉各项参数会由具体的操作执行人填入质卡 实例的检查项中, 若系统不具备参数库模块, 则操作工人 需频繁输入参数具体信息, 工作效率较低。同时, 该部分 数据分散在系统中, 车间管理人员无法掌握已有参数数据
整体情况, 产生数据资源浪费。由此可见, 参数管理对于 车间生产过程质量监控、提升车间管理水平至关重要。
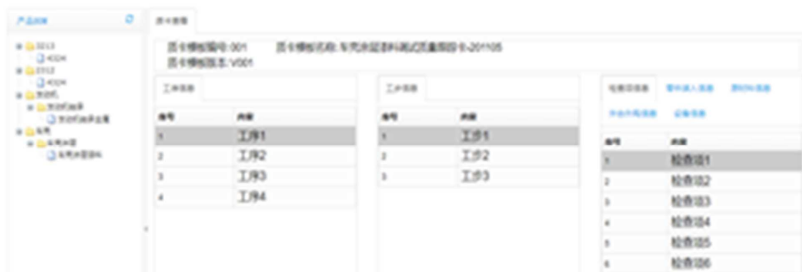

图3 质卡模板示例。

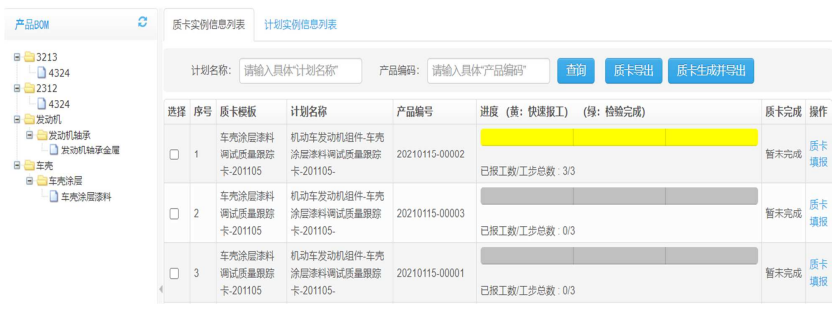

图4 质卡实例列表。

\section{2. 参数管理}

面向生产车间实际业务需求, 基于MES系统建立生产 过程参数库是实现参数管理的有效途径。同时, 对于参数 进行集中管理, 有利于生产车间工艺参数日常生产过程监 控及质量提升。下文将对参数管理所涉内容进行简要介绍, 主要包括参数特征、参数构成、参数管理模式等。

\subsection{1. 参数特征}

本文所涉参数为生产车间生产过程中零组件及成品 的各项工艺技术数据, 以产品质量跟踪卡为呈现实体, 记 录于质卡相应工步的检查项栏目下。产品参数主要包含: 参数编号、参数名称、产品名称、组件名称、取值区间、 区间属性、单位等信息，目前所涉及工艺数据均为结构化 数据, 暂不包含视频、音频等数据格式。表 1 为参数结构 示例, 因字段较多故仅选取部分字段进行展示。

表1 产品参数结构示例。

\begin{tabular}{lllllll}
\hline \multirow{2}{*}{ 产品参数结构 } & \multicolumn{5}{c}{ 参数属性 } \\
\cline { 2 - 7 } & 参数编码 & 参数名称 & 产品名称 & 组件名称 & 取值区间 & 区间属性 \\
\hline 1 & T1KJZP & 对称度 & Xxxsd & A组件 & $0-0.01$ & 闭区间 \\
2 & T1FZZP & 平行度 & Sddfj & A组件 & $0-0.002$ & 闭区间 \\
3 & T1CGQZ & 粗粘度 & Sdkjf & B组件 & $0-R a 0.4$ & 闭区间 \\
4 & T2SJOL & 质量差 & Klklj & C组件 & $200-240$ & 闭区间 \\
5 & T3SHJK & 接线柱高度 & Koeyu & K组件 & $3.65-3.8$ & 闭区间 \\
\hline
\end{tabular}

\subsection{2. 参数构成}

参数库中的工艺参数由文献手册参数及工程经验参 数两个主要部分构成。其中, 文献手册参数是指来源于国 际通用标准或业内通用文献中的标准数据集合; 工程经验 参数数据是源于本单位技术人员和操作工人的生产经验 积累, 比较符合生产实际, 但不一定最优, 且比较离散, 收集量较大 $[10]$ 。

\subsection{3. 参数管理模式}

参数管理模式由普通参数管理和参数集管理两部分 构成, 下文将对这两类参数管理模式进行简要介绍。

(1) 普通参数管理

普通参数管理主要实现了参数新增、删除、查找、升 级、导入、导出、参数集中展示等基本管理功能。该模块 具有严格的权限控制及流程管控，其中参数新增、删除、 升级仅能由工艺人员根据相关工艺规程发起申请, 经由工 
艺主管审批通过方可完成参数新增、删除、升级等操作。 系统进行了智能信息匹配, 数据导入时工艺人员只许提供 基础信息, 参数管理模块将根据产品名称及组件名称, 自 动查找对应BOM节点及质卡模板ID、工序、工步、检查 项等。

普通参数管理功能模块构成如图5所示。

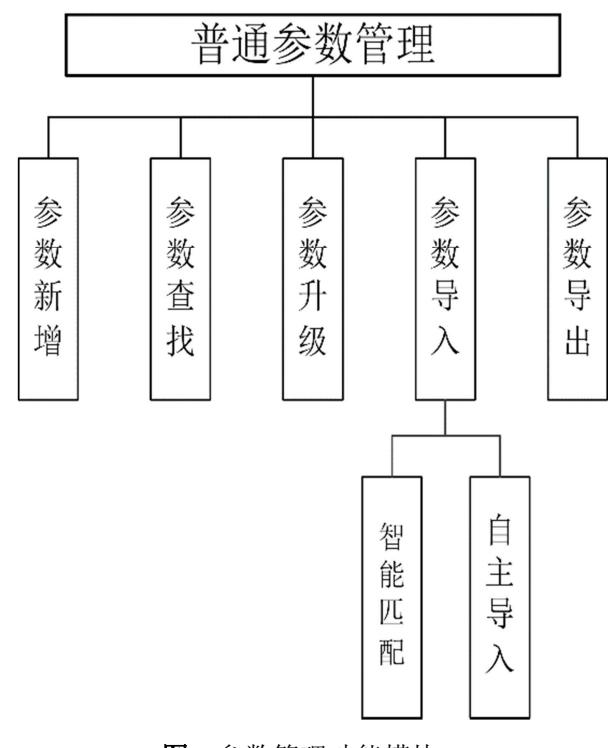

图5 参数管理功能模块。

(2) 参数集管理

同一质卡模板的所有检查项所涉参数统称为一个参 数集。参数集管理主要实现对于同一质卡下的工程经验参 数及文献手册参数的管理。参数集管理功能模块如图6所 示。除实现参数集分类展示、增、删、改、查、导入等基 本管理功能外, 还需完成数据分类、质卡关联及同步升级 操作。其中, 质卡同步升级是指当工艺人员完成质卡升级 后, 其对应的参数数据自动完成数据关联, 系统自动修改 参数对应的BOM、质卡模板ID等信息。与普通参数管理 相同的是, 参数集数据新增、删除、升级仅能由工艺人员 根据相关工艺规程发起申请, 经由工艺主管审批通过方可 完成。而与普通参数管理不同的是, 为提升操作人员工作 效率, 参数集参数新增、删除、查找、升级操作集成于质 卡模板的检查项页面, 操作人员可直接在质卡检查项页面 完成如上系列操作。

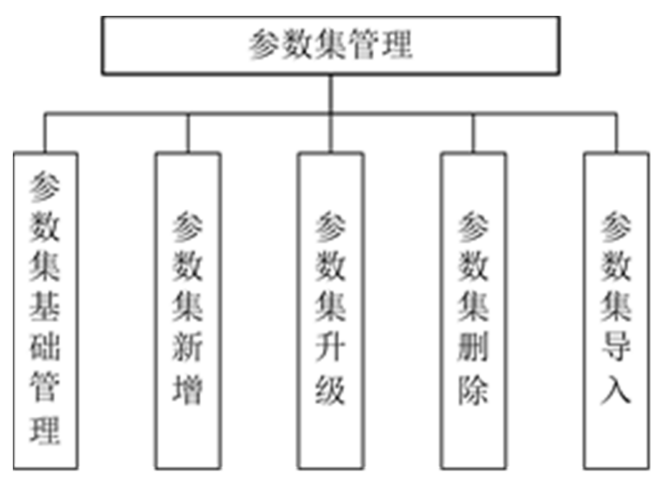

图6 参数集管理功能模块。
参数管理模块清晰展示生产过程所涉各项参数, 便于 工艺人员根据BOM或质卡等信息进行分类查找。而以参 数集方式进行管理, 是为了适用生产车间实际需求, 而提 出的与质卡相结合的创新管理方式, 方便操作工人实际填 报操作, 且无需重复进行信息输入, 提高工作效率, 同时 对于工艺人员来说方便进行生产车间工艺参数日常管理、 监控以及参数变更验证过程中的检查、跟踪。

\section{3. 参数优选}

参数优选流程如图7所示, 下文将对参数优选流程进 行详细说明。

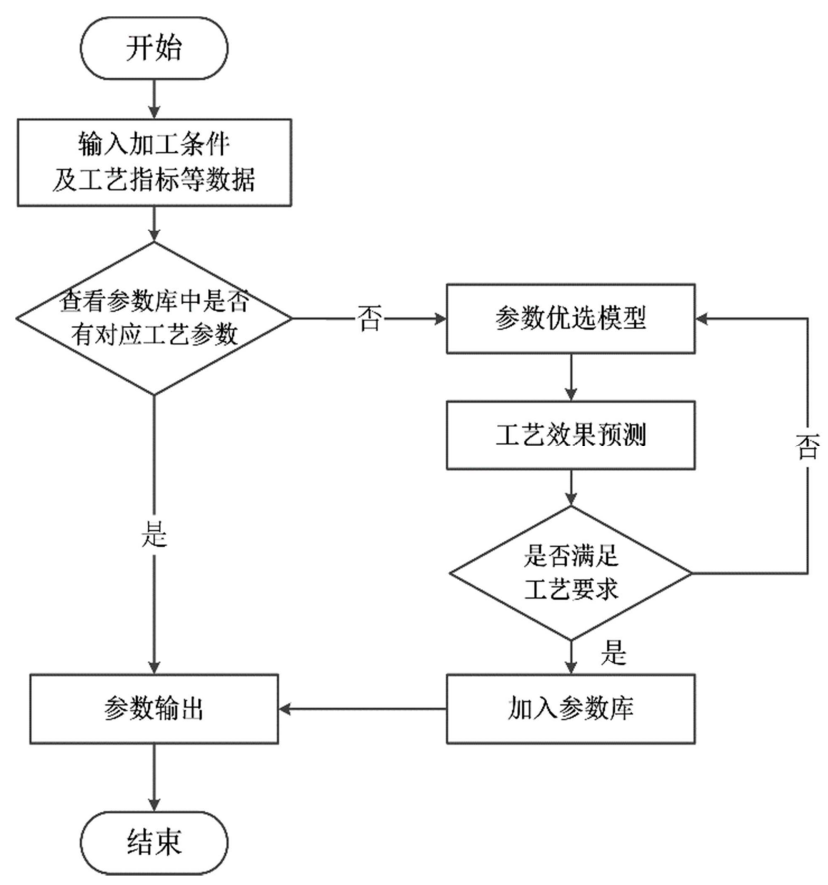

图7 参数优选流程。

工艺人员输入数据为工艺指标、加工条件, 系统根据 工艺人员输入数据, 在既有的参数库中进行分类查找, 若 能查找到满足要求的参数则完成参数选取。如果未发现满 足条件的参数, 随即将用户提供的工艺指标等数据输入至 参数优选模型, 后文将对该优选模型进行介绍。通过 BP 神经网络等算法进行参数优选, 对于优选后的参数进行工 艺效果预测, 如果能够满足实际应用即数据误差较小, 则 可以将该项参数加入至参数库中, 以备后续应用。

神经网络优选参数数据采用 $\mathrm{BP}$ 神经网络建模方法建 立组件加工工艺模型[11]。工艺模型如图8所示, 由于可能 涉及加工工艺技术细节, 故隐去模型实际输入、输出参数 名称。BP神经网络 (Back Propagation) 网络是一种多层 感知器, 包括输入层、中间层和输出层, 层与层之间的神 经元采用全互连的连接方式, 通过相应的网络权函数相互 连接; 每层内的神经元之间没有连接[12]。BP算法的基本 思想是, 学习过程由信号的正向传播与误差的反向传播两 个过程组成[13]。通过对样本数据进行优化选取, 不断地 将试验数据注入模型训练, 形成标准参数优选模式, 有效 解决加工工艺参数优选问题。 


\section{4. 参数填报及校验}

参数填报在质卡实例填报页面进行, 系统将根据参数 优选模型及工艺人员提供的有效参数完成参数优选, 为操 作人员提供参数范围, 同时在操作人员完成相关工作, 进 行参数填报时, 系统会自动完成参数范围校验并予以提醒。

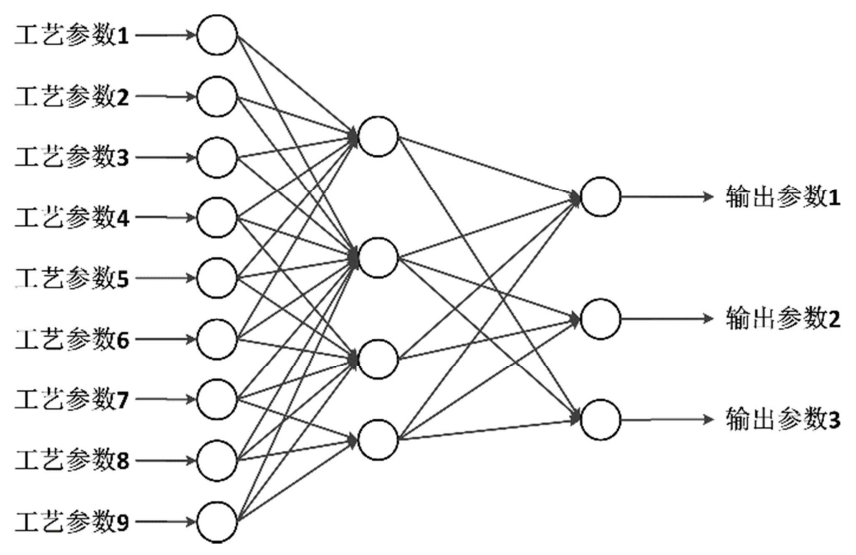

图8 工艺模型。

\section{5. 参数分析}

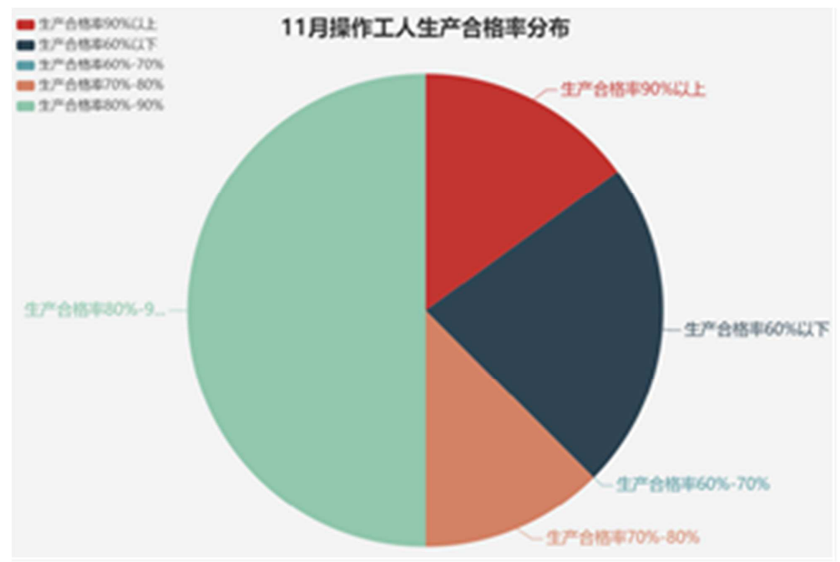

图9 11月操作工人生产合格率统计图。

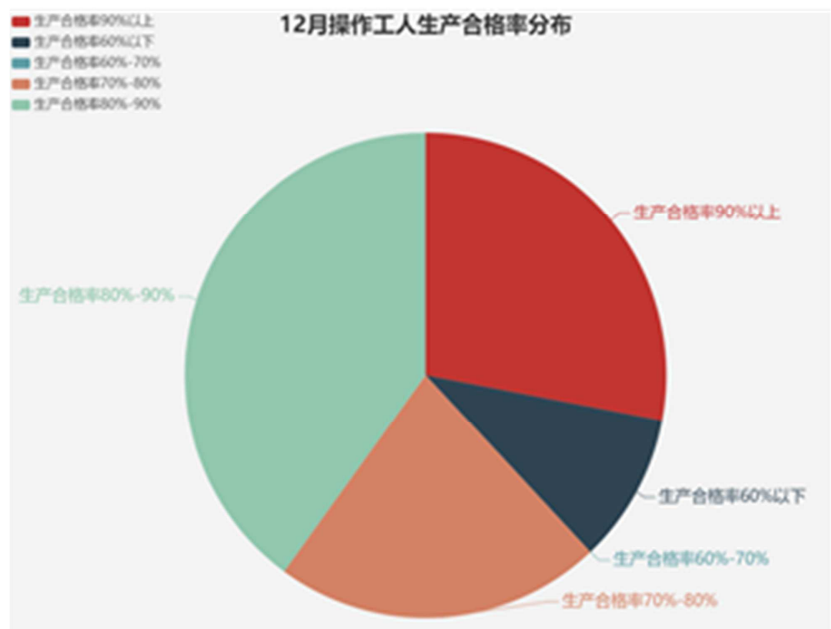

图10 12月操作工人生产合格率统计图。
历史加工过程中积累的参数数据对于指导今后的生 产加工过程及进行历史加工成果分析具有极大价值，依托 MES平台, 可以对已有参数数据进行统计分析, 以更为直 观的形式对于加工数据进行展示。同时, 车间生产质量管 理是生产车间信息化管理发展中至关重要的组成部分, 在 实际管理过程中，应灵活应用MES系统，全面的收集有关 的车间产品质量信息, 并经过详细的分析, 明确产品质量 的影响因素 [14], 对于生产过程质量控制意义重大。本节 将以某一组件的某项参数为研究对象, 选取 2020 年9月至 12 月生产车间所生产的 400 个同类型组件作为研究样本进 行参数分析。分析结果以图表形式展示, 如图9、图10、 图11、图12所示。

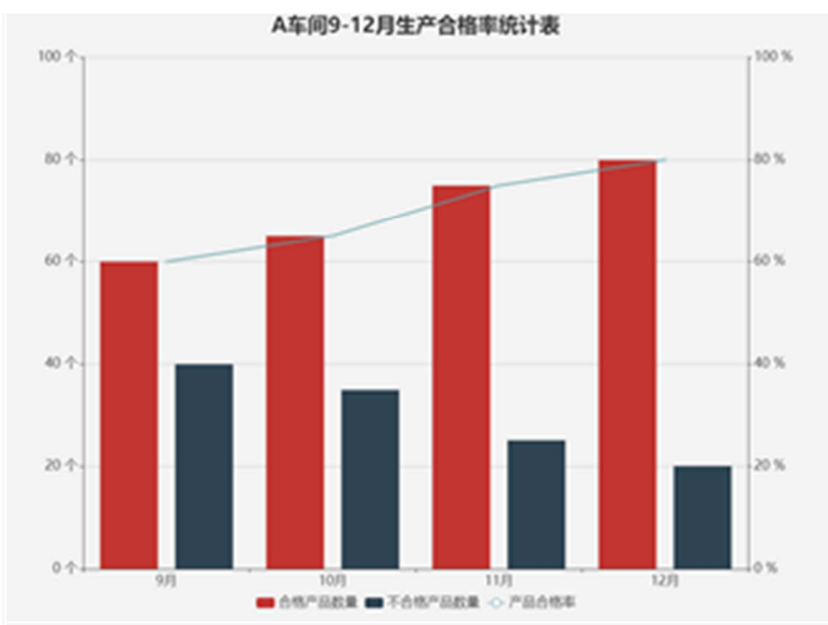

图11 A车间9-12月生产合格率统计表。

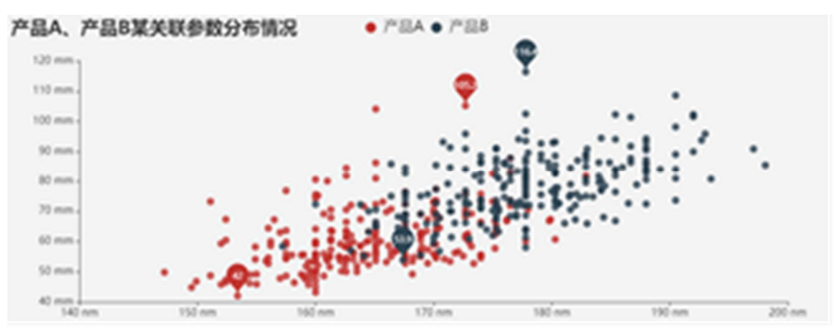

图12 产品A、产品B某关联参数分布情况。

通过上述系列统计图表, 我们可以得出以下结论:

1 、该车间 9 月- 12 月, 该组件合格产品共 280 件, 其中 9 月产品合格率为 $60 \%, 10$ 月产品合格率为 $65 \%, 11$ 月产品 合格率为 $75 \%, 12$ 月产品合格率为 $80 \%$ 。生产合格率总体 处于稳重有进状态。

2、该车间共 10 名操作工人进行该产品加工生产， 10 月、12月生产数据比较可以看出操作工整体生产合格率有 所提升。

3、产品 $\mathrm{A}$ 、产品 $\mathrm{B}$ 的参数 $\mathrm{X}$ 与参数 $\mathrm{Y}$ 均呈正相关。产 品 $\mathrm{A}$ 的参数 $\mathrm{X}$ 、参数 $\mathrm{Y}$ 均值均低于产品 $\mathrm{B}$ 。

实际生产过程中有效的参数分析对于生产过程质量 监控、质量回溯、操作工人成果量化对比具有重要参考意 义, 是参数管理至关重要的一个环节。通过参数分析模块, 可以给车间生产管理人员提供有力的决策支持, 继而采取 有效措施提高车间生产质量及管理水平。 


\section{4. 结语}

建立适合制造企业生产车间的参数管理机制, 对于提 升制造企业生产车间的产品质量、产品合格率、生产能力, 对于生产车间工艺参数日常管理及监控、主控参数变更的 提出、评估、验证、实施, 参数变更验证过程中的检查及 跟踪工作意义重大。

以MES系统为纽带, 打通领导、工艺人员、操作工人 之间的数字化通讯链路, 实现对生产过程实时管理及各项 生产参数的高效管理, 是生产车间保证产品加工质量和提 高生产加工效率的重要基础工程, 为生产车间信息集成、 数字化建设奠定了坚实的技术基础。同时, 也为企业生产 管理过程的持续改进、优化提供了有力的保障[15]。

\section{参考文献}

[1] 许红岩.浅谈MES系统在制造企业的应用[J].现代经济信息, 2019(10): 117.

[2] 肖宇亮.工业仪器仪表制造企业MES系统的设计与实现[D]. 湖北: 湖北工业大学, 2017.

[3] 耿育科.面向产品生命周期的BOM管理关键技术研究 [J].中 国技术新产品，2021(01): 122-124.

[4] 韦莎.智能制造系统架构研究 [J].信息技术与标准化, 2016(04): 50-54.
[5] 周子涵. MES系统在制造生产企业中的应用与研究[J].软件, 2020, 41(11): 214-216.

[6] 翁刚.基于MES系统的生产车间信息化管理探析[J].信息通 信, 2019(9): 138-140.

[7] 何芳霞, 余渊.电机质量跟踪信息系统解决方案和应用 $[\mathrm{J}]$. 上海大中型电机, 2012(03): 58-61.

[8] 孟友新, 黄帅, 张瑞全. 企业信息管理系统中产品质量跟 踪与追溯的研究与实现 $[\mathrm{J}]$, 微计算机应用, 2010(4) : 63-68.

[9] 袁家军.航天工程精细化质量管理 [J]. 中国工程科学, 2011(08): 36-42.

[10] 秦春涁. 数控铣削参数管理系统的研究 [J]. 矿山机械, 2012(5): 122-124.

[11] 蔡安江, 席洪波, 郭师虹. 电火花线切割加工参数智能自 选系统研究 [J]. 模具工业，2008，34(3): 69-72.

[12] LIU M S, CAO Z M, ZHANG J, et al.Short-term wind speed forecasting based on the jaya-SVM model $[\mathrm{J}]$. International Journal of Electrical Power and Energy Systems, 2020, 121(8), 106056.

[13] 蔡杰, 刘星潘, 炜冊, 靖轩. BP神经网络下的红外测温图 谱温度识别 $[\mathrm{J}]$.电子世界, 2021(05): 88-89.

[14] 周旭.基于MES系统的生产车间信息化管理研究 $[\mathrm{J}]$.电子世 界，2020(14)：59-60.

[15] 姚涛, 张忠波, 俞涛, 陈少平.船舶军工制造企业MES应用 研究 [J].现代制造工程，2017，(03)：31-37. 